\title{
INHALTSVERZE ICHNIS
}

Namenverzeichnis

Allgemeines:

Geschichte, Grundlagenwissenschaften, Jahresberichte, Statistik

Allgemeine Tierzucht:

Entwicklungslehre, Organe des Tierkörpers, Genetik, Fortpflanzung, Züchtung, Wachstum und Entwicklung, Ökologie einschließlich Haltung und Pflege, Nutzung und Qualität tierischer Erzeugnisse, Inhaltsstoffe tierischer Erzeug-

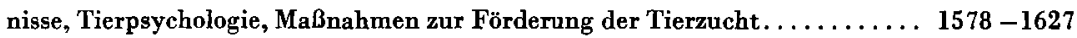

Spexielle GroBtierzucht :

Pferde, Rinder, Schweine, Schafe.

$1627-1654$

Spexielle Kleintierzucht :

Ziegen, Geflügel, Kaninchen, Pelztiere, Hunde, Bienen, Seidenraupen . . . . . . 1654 - 1677

\section{Tierernährung :}

Chemische Bestandteile des Tierkörpers und der Futtermittel, Untersuchungsund Versuchsmethodik, Ernährungsphysiologie, Verwertung der Nährstoffe und Futtermittel, Ernährungsstörungen und Mangelkrankheiten, Bewertung der Futterstoffe, Fütterung der landwirtschaftlichen Nutztiere........ $1677-1726$

\section{Futtermittelkunde:}

Futterstoffe, ihre Beeinflussung, Konservierung und Zubereitung, die einzelnen Futterstoffe, Misch- und Zusatzfuttermittel.

Fischerei :

Biologie, Lebensraum, Fischereiwirtschaft, Beeinflussung der Fischerei durch die Umwelt, Fischkrankheiten, Fischverarbeitung, Fanggeräte, Organisation und Planung, Fischereirecht, fischereiliche Wirtschaftsgeographie . . . 1753-1800

Buchbesprechungen $1625,1649,1789,1798,1800$

Bibliographische Angaben von Büchern und Dissertationen am SchluB eines jeden Kapitels 
NAMENVERZEICHNIS

Seitenzahl in [ K lammer] bedeutet Bibliographie, mit Stern * bedeutet Buchbesprechung

Aasen 1759 .

Abbott 1607 .

Abelein 1657

Achapkin 1616 .

Achinerov 1786.

Ackman 1791.

Adachi 1696 .

Adams 1716 .

Admina 1710 .

Adrianov 1616

Aitken 1660.

Ajzinbudas 1606

Akkermans 1664

tleandri 1608 .

Aleksiev 1595. 1652.

Alcxander 1689 .

Alipov 1735.

Alniquist 1776

Ambrosino 1620 .

Amman 1776 .

Anastassiadis 1592 .

Anderson 1585. 1707 .

Andersson 1711.

Andreev 1729.

Andres 1638 .

Andrews, E. D. 1712.

Andrews, J. D. 1789 .

Andrusevič 1611 .

Angelov 1744.

Anisimov 1727.

Antoni 1736.1741.

$$
1742 .
$$

Anwar 1719.

Aripdżanov 1662 .

Ariyama 1699.

Armstrong 1774 .

Arnold 1739 .

Asofsky 1591 .

Avdośev 1786 .

Babin 1616.

Babutia 1781 .

Bachmann 1682

Bährmann 1668 .

Bai 1685 .

Baid 1770 .

Bailey 1680.

Bajl'ozov 1790.

Baker, D. 1741.

Baker, H. K. 1730.

Băleanu 1777 .

Balevska 1608. 1725.

Balinsky 1766 .

Ball 1749 .

Balloun 1703.

Bal'ozov 1657

Balzer 1747.

Bank 1780. 1787.

Bańkivśkyj 1609.

Bankov 1715 .

Barber 1723.

Bargetzi 1758 .

Barker 1581 .

Barlett jun. 1604.

Barthelmes 1765 .

Bat'kaev 1726 .

Bayley 1696 .

Beames 1746.

Bedford 1598.

Bedö 1734.

Bedrata 1694.

Beeckler 1625.

Beker 1747 .

Belsare 1756.

Benda 1755.

Beremski 1646 .

Berenson 1680 .

Berger 1654.

Bergner 1733.
Bergströn 1714.

Berke 1734.

Bernhardt 1628.

Berry, F. H. 1762 .

Berry, J. E. 1595.

Bertrand 1738

Betina 1727.

Betzing 1683 .

Bhimachar 1775 .

Bianki 1756.

Bicri 1711.

Bindon 1594.

Birjuk 1580.

Biskai 1757 .

Bitteeva 1716 .

Bižev 1671. 1672.

Blau 1632.

Blaxter 1696.

Bloemendal 1688.

Blum 1712.

Böhme 1741 .

Boessneck 1579.

Bogner 1577.

Bolin 1729.

Bontuyan 1746.

Borek 1700 .

Borisov 1608.

Bosset 1765.

Bounia 1732 .

Bowman 1697.

Boyd 1757 .

Boyne 1718. 1738

Bradbury 1631.

Bramstedt 1678 .

Brandt, v. 1799 .

Branton 1738.

Braude 1723 .

Brauns 1652 .

Brazendale 1623 .

Bredon 1731. 1740.

Breitenstein 1795.

Breitfeld 1628.

Brennan 1690.

Bretschko 1668.

Briggs 1678.

Brinkhurst 1766.

Britov 1788.

Brodauf 1643.

Brodskij 1611.

Bröker 1667.

Bronsch 1618.

Brook 1766 .

Brown, K. I. 1603

Brown, L. D. 1705 .

Brüggemann 1705 .

Brümmergtädt 1796 .

Bruschek 1755.

Buchanan 1729.

Bǔčvarov 1676 .

Büttner 1799 .

Bugai 1778 .

Buhtz 1726.

Burdin 1737.

Burnham 1595.

Bursche 1769 .

Busch 1591.

Busnita 1783.

Bychowsky [1789]".

Calder 1723.

Campling 1694. 1708.

Capstick 1771.

Carius 1668.

Carlander 1753.

Carles 1690.

Carlson 1748.

Carrol 1690.

Carter 1652

Cassie 1772 .
Cassin 1692.

Castillo 1743

Castle 1737.

Catchpoole 1732 .

Cernobrovkin 1664 .

Certov 1782.

Cetyrkin, V.s. 1646.

Cetyrkin, V. V. 1646.

Chan 1699.

Chang 1598.

Chakraborty 1786.

Charnock 1701. 1709.

Chiesa 1688.

Chilson 1699.

C'hirilax 1771 .

Chlebnikov 1616 .

Christian 1590.

Cilingarjan 1600.

Cipera 1679.

Clamohoy 1746 .

Clapperton 1702.

Clausen 1750

Clegg 1599.

Clothier 1762.

Clugston 1797 .

Cole 1599.

Conneally 1591 .

Conner 1732.

Conway 1772

Cook 1590.

Coon 1720

Corbett 1738

Corriden 1785

Cotta 1781 .

Cowlishaw 1739 .

Cox 1592, 1647

Craig 1596.

Cranz 1736. 1741.

1742.1750.

Creutzberg 1757

Cristea, A. 1762. 1777. 1794.

Cristea, E. 1763. 1769.

Cristian 1760

Cronkite 1759.

Cross 1601.

Cuinat 1796.

Cukanov 1673.

Cumlivski 1651 .

Cunningham 1588.

Cupps 1589.

Curth 1674.

Cushing 1773.

Cvetkova 1619. 1620

Dachnovśkyj 1660 .

Dale 1578.

Dalferes jun. 1680 .

Dam 1706.

Damm 1614.

Danasoury 1731.

Darchen 1666 .

Darsie 1785

Das, B. R. 1681 .

Das, S. M. 1766.

Davidson 1718 .

Davies 1769 .

Davis 1625.

Davoren 1688.

Demark, van 1604 .

Demécenko 1786

Dennis 1603.

Denton 1752.

Derivaux 1599 .

Deufel 1781

Deyl 1683.

Dietrich 1790 .

Dijkgraaf 1757 .

Dikun 1790 .

Dimler 1677.

Dinusson 1729.

Uittrich 1577 .

Dobos 1708 .

Dobrev 1628.

Dobrynina 1592 .

bodsworth 1749 .

Domrałev 1616 .

Donev 1676. 1677.

Dorfman, A. S. 1585

Dorfman, R. I, 1585 . 1593.

Dorn 1658.

1)ougall 1722 .

Drăgăsanu 1768 .

Drawert 1682.

Orepper 1705.

Drysdale 1737 .

Ducos 1579

budarev 1617.

Dumitriu 1777.

Duyne 1698.

Džamusova 1767 .

Driuk 1596.

Dzjubenko 1609 .

Džurkov 1730 .

Eapen 1605

Eberhagen 1683 . 1751 .

Jibhardt 1628 .

Edwards 1593.

Eggum 1750.

Fgorov 1709.

Finsle 1766.

Gisenstein 1714.

El-Bolock 1787

Eliseev, J. T. 1795.

Eliseev, K. M. 1618 .

Elliot 1624 .

Ellich 1587

Emsanova 1790.

Engel 1655.

Ingelhard 1640 .

Engelmann 1656. 1724.

Englert 1665. 1666 .

Enkelmann 1681.

Entenman 1684.

Eppel 1661.

Erb 1597.

Erickson 1729 .

Eriksson 1711.

Esser 1740

Evang 1701 .

Evans, E. V. 1718 
Inthich 17?t.

Halcrow 1774.

Hall 1599 .

llalley 1722 .

Halsband 1784 .

Halverson 1730.

Hammer 1647 .

Hancock 1596 .

Hansen, A. Ji. 1720 .

Hansen, T). V. 1770.

Hansen, V. 1750 .

Haring 1715 .

Harms 1660.

Harper, A. T. 1703. 1704.

Harper, J. A. 1609.

Harper, N. 1619 .

Hart. J. I. 1712

Hart, P. ('. 1714

Harvey 1616

Hase 1678.

Iasek 1648.

Hass 1780 .

Hattop 1796

Haugse 1720.

Hauke 1621

Havel 1698 .

Hawkins 1792 .

Healy 1577 .

Heckl 1615

Hedin 1696 .

Heilinger 1728 .

Hellberg 1711 .

Hellström 1711.

Helm 1763

Hendrikse 1596.

Herbert 1784

Herrick 1586

Herrmann 1781 .

Herrmann, G. 1601

Herrmann, J. 1728

Hertelendy 1708 .

Hesselbach 1619

Hesselbarth 1745 .

Hetzel 1701.

Heywang 1749 .

Hill 1611.

Hilpert 1681 .

Hinds 1725.

Hinton 1586

Hoar 1757.

Hoare 1599.

Højgaard-Olsen 1750

Hörter 1585.

Hofer 1679 .

Hofmann, A. F. 1684

Hof $\operatorname{mann}, \mathrm{F} .1649$. 1656.

Hof mann, P. 1746.

Hoglund 1734.

Hohls 1748 .

Hole 1715.

Holleman 1725 .

Holmes, E. G. 1719 .

Holmes, W. 1738.

Holzschuh 1734

Horak [1643].

Horn, A. 1636 .

Horn, D. 1790 .

Howard, C. B. 1599.

Howard, D. A 1709 . 1737.

Howey 1622.

Hubbs 1785.

Hürzeler 1716 .

Hunt 1660 .

Hurley 1773.

Hurwitz 1660.

Hussain 1581.

Hutt 1724 .

Hvidsten 1715

Hyde 1738
Itomomos 1619. 1620. Kind 1608.

Il'ina 1726.

Imranov 1726.

Ingle 1683

fordache 1778

Iordănescu [1649]*.

Ipatenko 1612.

lrvancé 1609.

Ito 1578

Ivanov, I. F. 1714

Ivanov, P. 1641.

$$
1721 .
$$

Ivanova, I. M. 1791.

Ivanova, S. 1641

Izvorski 1646.

Jachinowic\% 1673. 1674.

Jacob 1639.

Jacquot 1712

Jagtenberg $17: 3$.

Jahn 1647.

James 1766 .

Janakiev 1673

Jangaard 1791 .

Janki 1729 .

Jansen 1681.

Jaroslav 1694.

Jclescu 1.783.

Jenes 1747 .

TCnš 1677

Jensen, I. S. 1729 .

Jensen, R. G. 1697

Jermolajeva 1694.

Johns 1698.

Johnson, J. B. 1597.

Johnson, R. R. 1748

Jones, F. R. H. 1793.

Jones, I. C. 1590 .

Jones, J. C. 1732.

Jones, J. W. 1764

Jones, R. W. 1677

Jonsson 1611.

Jordan 1674

Justice 1596 .

Kadijski 1640 .

Kaganovskij 1754

Kalkreuth, C. 1661 . 1662.

Kalkreuth, K. 1661 1662

Kállai 1708.

Kallen 1696.

Kalman 1672 .

Kalsbeek 1622 .

Kamar 1595.

Kan 1659.

Kaplan 1733 .

Karlov 1582.

Karlsson 1644 .

Karpenko 1786.

Karpov 1726. 1735 .

Kasdorif 1738 .

Katrandžiev [1625]*. Lamplin, 1598.

Kaufman 1710. $\quad$ Lampkin, K. 1709.

Kaufmann, $\mathbf{H}$. P.

$1685,1686$.

Kaufmann, W. 1736.

Kazakov 1606 .

Keetman 1784

Keiz 1754.

Kelleher 1700 .

Keller 1717

Kemmerer 1749 .

Kerényi 1785 .

Kessler 1766.

Kettner 1667. 1675 .

Ketz 1607. 1712.

Keyger 1705 .

Kiefer 1766

Kim 1714.

Kincl 1593.
Laitnerová 1644

Kinney 1710

Kirchgessner 1709.

iricenko 1581

irmse 1647.

irschke 162

Kirschner 1581 .

Kitto 1678

Klavins 1710.

lenk 1751.

Knape 1734 .

Knappen 1699.

Knauss 1771 .

Knjazev 1645

loop 1621 .

Kobashi 1678 .

Koch 1736 .

König, K.-H. 1653.

önig, L. 1629.

Kohlmeyer 1748 .

Koldaški 1646 .

Konrad 1621.

Konstantinov 1633.

Kormanovskaja 1644

Korostovecva 1577.

Kostin 1592.

Kostjunina 1704.

Kostomarov 1779 .

oval'skij 1710.

Kraack 1792.

Kramer 1690.

rampitz 1699

rause 1795

Kraut 1717

Krebs 1655 .

Krishnamurthy 1711 .

Kristensen 1706.

Krüger 1623.

ruse 1749 .

Kubu 1787.

Kulakovskaja 1786.

anov 1595. 1652. 1745 .

Kumta 1704.

unter 1621

Kunz 1773.

Kurjo 1793.

urnick 1725

Kuznecov 1644

Kuznecova 1616.

Kwa 1688.

Laird jun. 1722.

. 1598.

.

Langlands 1738 .

Langner 1766 .

Langston 1732 .

Lanigan 1732

Larsen 1792.

Larson 1730.

Lassiter 1705 .

Laube 1723. 1745. 1751.

Law 1706 .

Leat 1706

Laclercq 1599

Lecomte 1665 .

Le Cren 1762.

Leech [1643]
Kirov 1727. 1731.

Kolb 1587. 1679.

Leluwam 1733.

lei Ching 1786

lemburg 1600 ).

Lcnsky 1.666.

Leong 1729.

Jc Roy 16:35. 16:39.

Jewis 1696.1744 .

Liberatori 1620 .

Lie 1684

Liebenberg 1640 .

Liebmann 1754.

Ließ 1604.

Jillic 175 ?

Linke 1624.

Linzell 1582.

Mortimer 1754.

ittlcjohn J J 1785. Horton 1591 .

Littlejohn, $\boldsymbol{P}$. 1785. Mosolova 1733.

Livingstone jun. 1795. Moss 1755. 1756.

Lloyd 1784.

Lobmaier 1631

Löhle 1656.

Loen, van 1637.

Logvinovič 1782.

Lohniský 1758.

Irombard 1731 .

Lopatko 1605.

Lwenstein 1696 .

Ludwig 1712.

Lukov 1791.

Iund 1766 .

J,yhs 1654.

McArthur 1693.

McCarthy 1697.

IcCartney 1793.

MeClymont 1706 .

$\mathrm{McCullough} 1722$

McDaniel 1596 .

McDonald, C. E. 1682.

MacDonald, $T$. $H$. 1763.

MeFetridge 1618 .

McGibloon 1586 .

MeGinnis 1729 .

McGrew 1747.

Maclean 1631.

McNikoll 1678.

McPherson 1659.

IcShan 1586

Madsen 1750 .

Mäkelä 1638 .

Magakjan 1600 .

Magny 1690 
Pajin 1607.

Panajotov 1612.

Panajotova 1790 .

Pape 1798.

Pape, A. [1798]*.

Papenfui 1792.

Pappritz 1617.

Parker 1609.

Parsons 1615.

Pasvogel 1725.

Patel 1591.

Patrick 1659. 1707.

Patton 1697.

Paul 1798.

Pavlíéek 1683.

Pearcy 1787.

Pearsall 1754

Penner 1750.

icz 1669.1670 .

Peršina 1726.

Peters 1734.

Petersen 1623

Peterson 1625 .

Petkova 1608 .

Petrov 1713.

Pfeiffer 1644.

Phelps 1749.

Píanarosa 1620.

Piatkowski 1738.

Pickering 1585. 1707.

Pietro, di 1690.

Piggins 1757.

Pigon 1599 .

Pihl 1701

Pinfold 1618.

Pingel 1724.

Piskunov 1754.

Pljaščenko 1617.

Plonait 1720

Plotnikova 1735.

Podoba 1580.

Polin 1720.

Polkovnykova 1635.

Popenko 1726.

Popescu, E. 1768.

Popescu, F. 1695.

Popov, A. 1651.

Popov, I. 1672. 1727.

Popow 1666.

Porter 1720.

Porterfield 1591.

Pospeev 1584.

Postma 1615.

Postnikov 1727.

Power, B. A. 1678.

Power, R. F. 1678.

Prause [1798]*.

Preisler 1793.

Premachandra 1586. 1587.

Pressman 1687.

Pritsch 1675.

Proctor 1766.

Prusas 1661.

Ptáčk 1729.

Ptaškin 1709.

Püschel 1738.

Purves 1764.

Puškarskij 1630 .

Putnynja 1747.

Radu [1649]*.

Rădulescu 1788 .

Rahlmann 1589

Rajčev 1662

Ramaswamy 1701.

Randle 1697 .

Rangappa 1622 .

Rao 1581.

Rapoport 1590 .

Rass 1761

Rattray jum. 1770.
Reddy, B. S. 1742 .

Reddy, V. K. 1581 .

Reed 1579 .

Rees 1772.

Reichenbach-Klinke 1754.

Reid, B. L. 1725

Reid jun., J. L. 1771.

Reiske 1790.

Renatus 1614.

Reuther 1682

Richardson 1749.

Richter, K. 1736.

1741. 1742. 1750

Richter, W. 1656.

Riedel 1765 .

Riedmüller 1754 .

Rivas 1762

Rizvanov 1672

Rjabovič 1729 .

Robertson 1757.

Robinson 1744.

Rook 1708.

Rosca 1767. 1794.

Rose 1687.

Rosmus 1683 .

Rogs 1577. 1631

Rost 1686.

Rothe 1741.

Rott 1761.

Round 1766 .

Rudolph 1725 .

Rusoff 1738.

Ruttner 1667.

Ruževákyj 1636 .

Ružickij 1617.

Ruzskij 1634

Ryley 1736. 1745.

Sachova 1710.

Sacks 1751.

Sadylkov 1630

Saeki 1603.

Saff 1617 .

Saha 1767. 1786 .

Salgannik 1592.

Samachson 1692

Samoletov 1592.

Sanahuja 1703 .

Sanen 1759

Santamarina $\mathbf{1 5 9 8}$

Sapiro, E. A. 1767 .

Śapiro, I. M. 1761

Sasaki 1693

Sassaman 1690.

Sattler 1770.

Sauter 1625.

Saxena 1757 .

Ščkin 1629.

Schäfer, H. 1627.

Schäfer, W. 1603.

Schaumlöffel 1691 .

Schefold 1755.

Schell 1797

Scherr 1682

Scheuring 1769

Schiemenz 1763.

Schiller 1741. 1750 .

Schimmel 1679 .

sehmidt, K.-H. 1645.

$$
1742 .
$$

Sehmidt, W. 1734

Schmidt, W. J. 1770.

Sehmülling 1685 .

Schncider, K.-HI. 1659

Schneider, P. 1642

Schöps 1613.

Schröder. G. 1604.

Schröder, J 1743.

Schulpert 1715.
Scliulenlutirg 1603.

Schunm 1647.

Schussmann 1755.

Schwartz 1717.

Schwerin 1789.

Scott, H. M. 1703.

Scott, H. W. 1748

Sedgwick 1623.

Seher 1686.

Seidel 1587.

Sen, D. P. 1767. 1786

Sen, K. C. 1623 .

Sengel 1607

Sen Gupta 1767.

Senk 1721 .

Sensi 1688 .

Sentjakov 1796

Serpoianu 1771

Shin 1690 .

Sibbald 1718.

Siedler 1795.

Şiler 1645.

Silin 1720 .

Silvay 1690

Silver 1601.

Silverberg 1685 .

Simson 1791.

Sinclair 1718.

Singh 1756.

Sirotinin 1695

Sjöland 1610.

Skidmore 1684.

Skornjakov 1755

Skunkova 1747.

Skušek 1721 .

Slee 1652.

Slesarev 1747.

Smetnev 1656

Smišek 1782.

Smith, G. 1602

smith, J. J. B. 1763

Snctsinger 1703.

Soleanu 1767.

Someren, van 1754.

Sormmer 1799.

Sommerkamp 1615.

Sorenson 1581 .

Soulsby 1777.

Southward 1773.

Spais 1709.

Spencer-Laszlo 1692

Splittgerber 1744 .

1745. 1746 .

Squires 1762 .

Srivastava 1766.

Stajkov 1670.

Starkova 1709 .

Staroverov 173:3.

Stascheit 1791.

Steger 1738

Stehle 1672.

Steklenev 1651

Stelos 1687 .

Stengel 1606.

Stenius 1590.

Steopoe 1761.

Stephenson, E. L. 1751.

Stephenson, L. K. 1607.

Sternkopf 1680 .

Stevenson 1586

Stewart 1772.

tichmann 1626.

Stoev 1677.

Stohl 1713.

Stoina 1768 .

Stone 1591 .

stormshak 1597 .

Št'ota 1785.

Sturkie 1583. 1594

1705 .

Suceveanu 1788.

sugital 1701 .

sullivan 1716. 1725. Venkatayan 1581.

Susai 1760 .

Suševyč 1694

Svišcova 1694

Swarup, H. 1756

Vinson 1590

Swell 1706.

Swingle 1753.

Tacu 1695 .

Tambovcev 1584.

Tanev, D. 1652 .

Tanev, I. 1651.

Tarján 1708.

Tarrant 1622 .

Taylor, L. R. 1577.

Taylor, T. G. 1708 .

Teal 1774.

Tembrock 1626.

Templeman 1762 .

Teodorescu-Leonte 1761

Terpstra 1664.

Tertiscu 1782.

Textor 1583.

Thibault 1596.

Thiel 1773.

Thiele 1640 .

Thomas 1742 .

Thomke 1741. 1743.

Thorbecke 1591 .

Thornburg 1689.

Thorsøe 1593 .

Thulke 1653.

Tichomirov 1714

Tillman 1749 .

Timiras 1582.

Tockaja 1580 .

Todd 1627.

Todorov 1619. 1620.

Tokuyasu 1687.

Toman 1785 .

Tomaschko 1755 .

Tomov, I. 1735 .

Tomov, V. 1658.

Tonna 1759 .

Topliff 1792 .

Topps 1723. 


\section{LANDWIRTSCHAFTLICHES ZENTRALBLATT ABT. TIERZUCHT·TIERERNÄHRUNG · FISCHEREI}

\section{ALLGEMEINES \\ A. GESCHICHTE, FORSCHUNG, UNTERRICHT}

Ross, K.; Halle/S. Martin-Luther-Univ. Halle-Wittenberg, Inst. für Tierzucht. Professor Dr. Werner Wussow 60 Jahre alt. Arch. Tierzucht 6 (1963) Nr. 4. S. $249-250$.

Bogner, H. Aufgaben und Lrgebnisse einer Tierzuchtversuchsanstalt, dargestellt am Beispiel der Bayerischen Landesanstalt fiur Tierzucht in Grub. Schweiz. landwirtsch. Mh. 41 (1963) Nr. 1. S. 3-30. - 9 Tab.

Das 1918 aus Mitteln der Bauernschaft angekaufte Gut Grub sollte durch Versuchsanstellungen sowie durch Ausbildung und Schulung von Fachkräften die Leistungsfähigkeit der landwirtsch. Nutztiere fördern. 1940 übernahm das Bayerische Landwirtschaftsministerium das Institut mit der Bezeichnung „Bayerische Landesanstalt für Tierzucht". Der Ausbau erfolgte nach 1945 durch Z orn. Die Anstalt hat vorwiegend folgende Aufgaben zu erfüllen: 1. Versuche und Forschungen, die auf die praktische Nutzanwendung aus. gerichtet sind. 2. Durchführung von Lehrgängen. 3. Veröffentlichungen und Vorträge. 4. Durchführung von Leistungsprüfungen. - Die Versuchsergebnisse sowie die laufenden Forschungsvorhaben werden angeführt.

H. Kleiber. 40.

Dittrich, W. Weitere Entwicklung des sozialistischen Bildungswesens in der Milchindustrie. Dtsch. Milchwirtsch. 10 (1963) Nr. 2. S. 54-56.

Das Ausbildungswesen der Milchwirtschaft hat sich den neuen veränderten Produktionsbedingungen anzupassen. Hierauf ist bereits im polytechnischen Unterricht einzuwirken. In den polytechnischen Oberschulen können die Grundlagen in der Berufsausbildung für verwandte Berufsgruppen gelegt werden. - Über die spez. Ausbildung von Laboranten in Dresden und Güstrow, Assistenten und Ingenieuren in Halberstadt, für Meister in Güstrow und für Diplom-Milchwirte an der Humboldt-Univ. Berlin und deren Ausbildungsziele wird berichtet. Bes. Augenmerk wird auf kombinierte Fernstudien mit laufend steigendem Qualifikationsniveau gelenkt. Für 1963 ist die Erarbeitung eines neuen Studienplanes vorgesehen, nach dem sich der Ausbildungs. gang org. vom Facharbeiter bis zum akademischen Kader gliedert.

W. Zeller. 50 .

\section{B. GRUNDLAGENWISSENSCHAFTEN UND METHODIK}

Korostoveeva, N. V. (Korostowzewa. N. W.); Leningrad, Inst. für Bluttransfusionen, Abt. für Exp. Pathol. $\mathbf{k}$ metodike iskusstvennogo dychanija $\mathrm{v}$ opytach na melkich životnych. (Zur Methodik der kuinstlichen Beatmung bei Versuchen mit Kleintieren.) Fiziologičeskij žurnal SSSR imeni I. M. Sečenova, Moskva-Leningrad 49 (1963) Nr. 2. S. 260-261. - 1 Abb. 120.

Healy, M. J. R. und Taylor, L. R. Tables for power-law transformations. (Tafeln für Potenztransformationen.) Biometrika [London] 49 (1962) Nr. 3/4. S. 557-559. - 8 Tab., 3 Lit.

Beobachtungsdaten müssen oft transformiert werden, bevor eine statistische Auswertung vorgenommen werden kann. Neben $z=\log x$ kommt der Trans- 